\title{
Temperature dependence of the surfactant film bending elasticity in a bicontinuous sugar surfactant based microemulsion: a quasielastic scattering study $\dagger$
}

\author{
Stefan Wellert, ${ }^{* a}$ Matthias Karg, ${ }^{b}$ Olaf Holderer, ${ }^{c}$ André Richardt ${ }^{d}$ and \\ Thomas Hellweg ${ }^{e}$
}

Received 5th October 2010, Accepted 18th November 2010

DOI: $10.1039 / \mathrm{c0cp02044c}$

Currently, the design of microemulsions is focussed on the formulation of environmentally compatible systems formed by non-harmful amphiphiles and oils. The use of sugar-based surfactants allows the design of microemulsions where, instead of the temperature, the addition of short- or medium-chain alcohols tunes the curvature of the amphiphilic interface. In this work, the resulting temperature stability of a sugar surfactant and rapeseed methyl ester based bicontinuous microemulsion is exploited to study the influence of temperature variations on the bending elastic constant $\kappa$. Quasi-elastic scattering of light and neutrons is used to separate long-range collective motions and local thermally excited undulations of the interface. $\kappa$ in units of $k T$ is found to be independent of temperature over a wide range.

\section{Introduction}

Biomass based surfactants gain importance for many applications due to excellent environmental compatibility and lower toxicity compared to established surfactants. A prominent example for this group are sugar surfactants i.e. alkyl oligoglucosides $\left(\mathrm{C}_{m} \mathrm{G}_{n}\right)$. Their phase behaviour is of particular interest, since the behavior of the well known nonionic surfactants of the alkyl oligo ethyleneoxide group $\left(\mathrm{C}_{i} \mathrm{E}_{j}\right)$ can be reproduced using the addition of alcohol to tune the natural curvature of the surfactant film. ${ }^{1}$ The main difference between the two classes of nonionic surfactants is the low temperature dependence of the phase behaviour of sugar surfactant based pseudo ternary systems. ${ }^{2,3}$ Due to the strength of the hydrogen bonds between the hydroxy groups in the sugar headgroup and water no significant dehydration of the headgroup is observable with increasing temperature, ${ }^{4}$ but instead the phase behaviour can be controlled using the addition of alcohols. ${ }^{5}$

${ }^{a}$ Institut für Chemie, Technische Universität Berlin,

Strasse des 17.Juni 124, D-10623 Berlin, Germany.

E-mail: s.wellert@tu-berlin.de

${ }^{b}$ Bio21 Institute and School of Chemistry, University of Melbourne,

30 Flemington Rd., 3010 Victoria, Australia

${ }^{c}$ Jülich Center for Neutron Scattering, FRM II Garching,

Lichtenbergstr. 1, D-85747 Garching, Germany

${ }^{d}$ Wehrwissenschaftliches Institut, für Schutztechnologien - ABC-Schutz, Humboldtstrasse, D-29633 Munster, Germany

${ }^{e}$ Universität Bielefeld, Physikalische und Biophysikalische Chemie (PCIII), Universitätsstraße 20, D-33615 Bielefeld, Germany.

E-mail: thomas.hellweg@uni-bielefeld.de

$\dagger$ This article was submitted as part of a special collection on scattering methods applied to soft matter, marking the 65th birthday of Professor Otto Glatter.
This property is important for a series of applications. For example, within optimized reaction systems the temperature can be used as additional degree of freedom to tune the reaction kinetics of enzymes or catalytic particles. Furthermore, it provides a greater flexibility with respect to applications under changing environmental conditions, e.g. if the external parameter temperature is not constant.

From a physical chemistry point of view sugar surfactants are interesting, because they allow the study of some aspects of microemulsions, which were not accessible with the well known $\mathrm{C}_{i} \mathrm{E}_{j}$ surfactants. In particular, since a temperature variation does not significantly alter the phase structure and its structural properties, it is possible to study thermally excited fluctuations of the interface in a comparatively wide temperature range, corresponding to activation energies of stochastic motions of the interface. Experimentally, these fluctuations can be studied with neutron spinecho spectroscopy (NSE), ${ }^{6}$ a technique, which measures the normalized intermediate scattering function in the pico- to nano-second time window, typically in a $q$ range between $10^{-2} \AA^{-1}$ up to a few $\AA^{-1}$. Depending on the Fourier time window and the $q$-range explored in the measurements beside local motions and their typical $q^{3}$ dispersion relation of the relaxation rates, also long range collective motions with low relaxation frequencies linear in $q^{2}$ were observed. ${ }^{7-11}$ In some of these cases the slow mode is related to center of mass diffusion, but also bicontinuous systems exhibit slow, diffusional, collective modes. ${ }^{12}$

Dynamic light scattering (DLS) is another quasielastic scattering technique, which provides the possibility to explore collective dynamics inside mesoscopically structured complex 
Table 1 Temperature dependence of density, kinematic and dynamic viscosity of RME. The dynamic viscosity was calculated according to $\eta_{\mathrm{RME}}=\nu_{\mathrm{RME}} \times \rho_{\mathrm{RME}}$. For comparison, the corresponding values of the dynamic viscosity of $\mathrm{D}_{2} \mathrm{O}^{31}$ are added

\begin{tabular}{lllll}
\hline$T / \mathrm{K}$ & $\nu_{\mathrm{RME}} / \mathrm{mm}^{2} \mathrm{~s}^{-1}$ & $\rho_{\mathrm{RME}} / \mathrm{g} \mathrm{cm}^{-3}$ & $\eta_{\mathrm{RME}}(\mathrm{mPas})$ & $\eta_{\mathrm{D}_{2} \mathrm{O}}(\mathrm{mPas})$ \\
\hline 283 & 10.378 & 0.890 & 9.244 & 1.543 \\
293 & 7.350 & 0.881 & 6.472 & 1.244 \\
298 & 6.645 & 0.877 & 5.827 & 1.127 \\
302 & 5.729 & 0.873 & 5.003 & 1.021 \\
\hline
\end{tabular}

fluids exhibiting characteristic lengths up to a few hundred nanometres. In the literature only few publications report on the dynamics of similar samples like polymeric bicontinuous microemulsions, ${ }^{13}$ concentrated droplet phases, ${ }^{14}$ lamellar and sponge phases ${ }^{15-18}$ using dynamic light scattering experiments. In almost all of these works two relaxation modes were observed. In all cases the found relaxation rates showed a dispersion relation, which was linear in $q^{2}$. This is typical for diffusive modes.

In addition to DLS and NSE also small angle scattering can be used to study the structure and the correlation length of microemulsions. ${ }^{19-22}$ According to Pieruschka and Safran ${ }^{23,24}$ the renormalized bending elastic constant can be obtained from SAXS or SANS data. This quantity can be converted into the bending elastic constant accessible by quasielastic scattering techniques. ${ }^{8}$

In the following, we report a study of a bicontinuous microemulsion system formed by the environmentally compatible oil component rapeseed methylester (RME) and a technical grade sugar surfactant. The system was designed as new carrier medium for the decontamination of toxic lipophilic compounds, e.g. organophosphates like chemical warfare agents or pesticides $^{25}$ from hydrophobically varnished solid surfaces. Such a carrier medium has to extract the water insoluble toxic compound and to serve as a reservoir for a chemical decomposition via the amphiphilic interface, e.g. by enzymatic hydrolysis. ${ }^{26}$ We use quasielastic scattering methods to investigate the influence of the sample temperature on the dynamics of the interfacial film, in particular on the bending elasticity constant $\kappa$ as used in the theoretical description of the curvature free energy. ${ }^{27}$

As mentioned above, local dynamics and collective dynamics both can be observed in NSE measurements and for a reasonable analysis it is necessary to separate both modes. ${ }^{10}$ Different approaches were developed to overcome this problem. In this work, a combination of NSE and dynamic light scattering (DLS) measurements is used to identify both types of contributions to the NSE signal by including additional measurements of the normalized intensity time autocorrelation function $g^{2}(\tau)$ in the $q$-range $q \ll q_{\max }$, where $q_{\max }$ represents the maximum of the static structure factor $\mathrm{S}(q) .^{10,28,29}$

The results from the quasielastic scattering experiments will be compared to data derived from SAXS measurements.

\section{Experimental}

\subsection{Materials}

The amphiphilic film of the microemulsion system under investigation consists of the technical grade sugar surfactant SL55, a $\mathrm{C}_{12 / 14} \mathrm{G}_{1.3}$ alkyl oligoglucoside (Seppic GmbH,
Germany) and Pentanol (Sigma Aldrich). Commercially available rapeseed methyl ester (RME, biodiesel) was purchased from a local gasoline station and used as oil component. The biodiesel used in the experiments was produced in accordance with the European standard EN 14214 and consists of a mixture of saturated and unsaturated fatty acid methyl esters, $\mathrm{CH}_{3}$-OOC-R. $\mathrm{R}$ represents the fatty acid component with a chain length distribution from $\mathrm{C}_{16}$ to $\mathrm{C}_{24}$ and with $80-90 \%$ the $\mathrm{C}_{18}$ is the main component in this mixture. ${ }^{30} \mathrm{D}_{2} \mathrm{O}$ was received from Eurisotop (France) (isotopic purity $\geq 99 \%$ ). Further information about the components, particularly about the RME can be found in the literature. ${ }^{30}$

\subsection{Determination of sample viscosity}

Knowledge of the dynamic viscosity of the bulk phases is necessary for the determination of the bending elastic constant as described in section 3.3. The temperature dependence of the dynamic viscosity $\eta_{\mathrm{D}_{2} \mathrm{O}}$ of $\mathrm{D}_{2} \mathrm{O}$ was taken from the literature. ${ }^{31}$ Since the composition of different RME charges can vary, the required physical properties have to be experimentally determined. According to $\eta_{\mathrm{RME}}(T)=\nu_{\mathrm{RME}}(T) \times \rho_{\mathrm{RME}}(T)$ from measurements of the density $\rho_{\mathrm{RME}}$ and the kinematic viscosity $\nu_{\mathrm{RME}}$ the dynamic viscosities $\eta_{\mathrm{RME}}$ of rapeseed methyl ester was calculated. Density measurements were done using a DMA 4500 densitometer (Anton Paar $\mathrm{GmbH}$, Germany) and the kinematic viscosity was determined using an Ubbelohde viscosimeter (Schott, Germany). Table 1 summarizes the results for RME, the calculated dynamic viscosity and the corresponding dynamic viscosities of $\mathrm{D}_{2} \mathrm{O}$.

\subsection{The microemulsion system}

Fig. 1 shows a Kahlweit-fish type cut through the phase tetrahedron of the quaternary microemulsion system water/ $\mathrm{RME} / \mathrm{C}_{12 / 14} \mathrm{G}_{1.3} /$ Pentanol. The phase behavior was studied at a constant temperature of $298 \mathrm{~K}$ using a fixed weight fraction $\alpha=0.5$ of the oil in the mixture of water and oil. ${ }^{32}$ A detailed discussion of the phase behaviour can be found in ref. 30. At a surfactant content of $\gamma=0.26$ and an alcohol content of $\delta=0.07$ the one-phase region starts to form. The composition of the investigated bicontinuous sample at $\alpha=0.5, \gamma=0.299$ and $\delta=0.07$ is indicated in the phase diagram (see Fig. 1). From previous SANS measurements and a Teubner-Strey analysis of these data a mean domain size d of $130 \AA$ and a correlation lengths of 44-47 $\AA$ were obtained. ${ }^{30}$ A stock solution was prepared by weighing in and stirring appropriate amounts of each component. The scattering experiments at

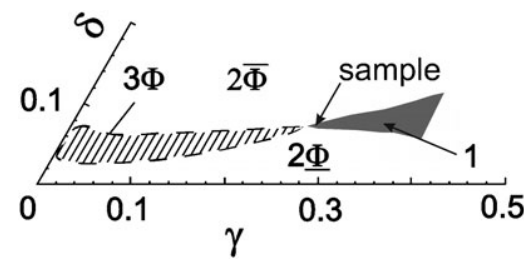

Fig. 1 The phase diagram of the quaternary system water-RME$C_{12 / 14} G_{1.3}$-Pentanol in a Kahlweit-fish type cut. ${ }^{53}$ Shown are the threephase body, the two-phase regions above and below this body and the one-phase region in the fish tail. The location of the sample composition point is indicated. 
different temperatures reported here, were performed with new portions from the homogeneous microemulsion stock solution. Prior to each measurement, the samples were thermalized at the desired temperature in a thermostated bath and transferred into a previously thermalized sample holder system.

\subsection{Small angle X-ray scattering (SAXS)}

The SAXS measurements were performed using a SAXSess $\mathrm{mc}^{2}$ system (Anton Paar KG, Graz, Austria) equipped with a sealed tube microsource having in line collimation, operated at $40 \mathrm{kV}$ and $50 \mathrm{~mA}$ producing $\mathrm{Cu}-\mathrm{K} \alpha$ radiation having a wavelength of $0.154 \mathrm{~nm} .{ }^{33}$ Inital data were treated using the Saxsquant 3.5 software package. Data were corrected for the dark current and scattering from the blank cell by subtracting the contributions. Desmearing was achieved by including the measured beam length profile in the desmearing procedure. The data were brought to absolute scale by using the constant scattering intensity of water as a secondary standard. ${ }^{34}$ The sample was measured in a $1 \mathrm{~mm}$ quartz capillary and equilibrated for $30 \mathrm{~min}$ at the desired temperature. The scattering curves were analyzed by fitting the Teubner-Strey model to the data. The SASfit software (by J. Kohlbrecher from the Paul Scherrer Institute, Villigen, Switzerland) provides a corresponding non-linear least square fitting routine.

\subsection{Dynamic light scattering}

Dynamic light scattering measurements were performed with a goniometer setup (ALV Langen, Germany) equipped with pinhole collimation, frequency doubled Nd:YAG laser (Compass series, Coherent, USA) of a nominal power of $150 \mathrm{~mW}$ at $\lambda=532 \mathrm{~nm}$. Intensity time correlation functions were generated using a multiple- $\tau$ correlator ALV-5000/E (ALV GmbH, Langen, Germany). The samples were filtered with an Anotop filter of pore size $1 \mu \mathrm{m}$ to remove dust and large impurities prior filling into cylindrical Hellma quartz cells of $1 \mathrm{~cm}$ outer diameter. In addition to measurements in standard cells also NMR quartz tubes were used to provide a shorter optical path inside the sample cell to test for multiple scattering effects. A comparison of the relaxation rates obtained from these measurements at scattering angles of $75^{\circ}, 90^{\circ}$ and $100^{\circ}$ shows a relative deviation of $0.3 \%$ to $0.5 \%$. Therefore, multiple scattering effects can be neglected here. Prior to the measurements all samples were thermalized at the desired temperature. The measured normalized intensity autocorrelation functions were analyzed using the inverse Laplace transformation algorithm implemented in the program CONTIN. ${ }^{35}$

\subsection{Neutron spin-echo spectroscopy}

NSE experiments were carried out at the J-NSE instrument ${ }^{36,37}$ at the FRMII neutron source in Garching, Germany. All samples were measured at five scattering angles corresponding to momentum transfer values $q=0.05 \AA^{-1}, q=0.08 \AA^{-1}$, $q=0.11 \AA^{-1}, q=0.15 \AA^{-1}$ and $q=0.18 \AA^{-1}$ using neutrons of wavelengths $\lambda=8 \AA$ and $\lambda=12 \AA$. The samples were thermostated at the desired temperatures. During the NSE measurements a second identical sample was kept in a thermostated bath at the desired temperature to monitor the long time stability of the phase behaviour. Measurements were

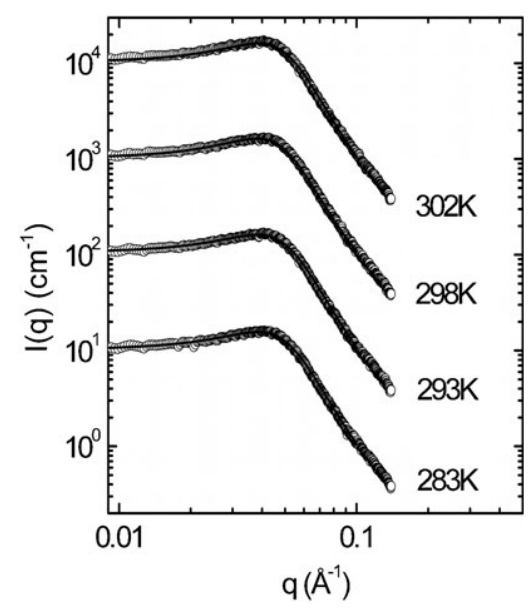

Fig. 2 Experimental SAXS curves of a bicontinuous sample measured at four sample temperatures after background subtraction and absolute scaling using water as a secondary standard. The curves were shifted with respect to each other by multiplication with multiples of ten for better visualisation. Solid lines are fits according to the Teubner-Strey model. The results of the analysis are summarized in Table 2.

done at four sample temperatures $283 \mathrm{~K}, 293 \mathrm{~K}, 298 \mathrm{~K}, 302 \mathrm{~K}$. The resolution function $\mathrm{S}(q, 0)$ of the instrument was measured using graphite and mesoporous silica material.

\section{Results and discussion}

\subsection{Determination of $\boldsymbol{\kappa}$ from SAXS}

Fig. 2 shows the background corrected desmeared SAXS curves of a bicontinuous sample from the bicontinuous microemulsion. The curves were recorded at different sample temperatures (see labels in Fig. 2). The broad structure factor maximum is the characteristic feature of a bicontinuous structure. Solid lines are fits of the Teubner-Strey model using the corresponding non-linear least square routine implemented in the SASfit software. ${ }^{38}$ The Teubner-Strey approach results from an order parameter expansion of the free energy associating the water-to-oil ratio inside the microemulsion with the order parameter $\psi$. In ref. 19 it was shown that a coefficient combination $a_{i}=0$ except $a_{2}>0$ and $c_{i}=0$ except $c_{1}<0$ and $c_{2}>0$ results in a Landau free energy $F$ of the form

$$
F=\int\left(a_{2} \psi^{2}+c_{1}(\nabla \psi)^{2}+c_{2}(\Delta \psi)^{2}\right) \mathrm{d}^{3} r
$$

with the stability condition $4 a_{2} c_{2}-c_{1}^{2}>0$. This leads to a scattered intensity distribution given by

$$
I(q)=\frac{8 \pi / c_{2}\left\langle\eta^{2}\right\rangle V \xi_{T S}}{a_{2}+c_{1} q^{2}+c_{2} q^{4}}
$$

Table 2 Correlation length $\xi_{T S}$ and domain size $\mathrm{d}_{T S}$ obtained from fits to the SAXS curves plotted in Fig. 2 according to the TeubnerStrey model. Additionally, the renormalized bending elasticity constant $\kappa_{\text {SAXS }}$ is listed

\begin{tabular}{lllll}
\hline$T / \mathrm{K}$ & $\xi_{T S} / \AA$ & $d_{T S} / \AA$ & $\kappa_{\mathrm{SAXS}}\left(k_{\mathrm{B}} T\right)$ & $\kappa^{\text {theo }}\left(k_{\mathrm{B}} T\right)$ \\
\hline 283 & 43.7 & 140.5 & 0.26 & 0.57 \\
293 & 44.0 & 141.2 & 0.27 & 0.57 \\
298 & 47.0 & 144.2 & 0.28 & 0.58 \\
302 & 45.0 & 141.0 & 0.27 & 0.57 \\
\hline
\end{tabular}


The expansion parameters $a_{2}, c_{1}, c_{2}$ allow the determination of the mean domain size $d_{T S}$ of the structure

$$
d_{T S}=2 \pi\left(\frac{1}{2}\left(\frac{a_{2}}{c_{2}}\right)^{1 / 2}-\frac{c_{1}}{4 c_{2}}\right)^{-1 / 2}
$$

Furthermore, the correlation length $\xi_{T S}$ can be calculated according to

$$
\xi_{T S}=\left(\frac{1}{2}\left(\frac{a_{2}}{c_{2}}\right)^{1 / 2}+\frac{c_{1}}{4 c_{2}}\right)^{-1 / 2}
$$

and describes the length scale of the intermediate range fluctuations. The fit leads to the $\xi_{T S}$ and $d_{T S}$ values given in Table 2. In the investigated temperature range of $20 \mathrm{~K}$ no significant change in domain size or correlation length was observed within the accuracy of the experiment.

According to Pieruschka and Safran ${ }^{23}$ the renormalized bending elastic constant $\kappa_{\text {SAXs }}$ can be calculated from the correlation length $\xi_{T S}$ obtained from the Teubner-Strey analysis of small angle data using ${ }^{39}$

$$
\frac{\kappa_{S A X S}}{k T}=\frac{10 \sqrt{3} \pi}{64} \frac{\xi_{T S}}{d_{T S}}
$$

Using the fit results the renormalised $\kappa_{\text {SAXS }}$ values are computed for the different temperatures.

$\kappa_{\text {SAXS }}$ comprises membrane fluctuations up to the characteristic length scale of the bicontinuous microemulsion. From neutron spin echo spectroscopy a bare bending elasticity constant $\kappa$ can be determined, which is different from $\kappa_{\text {SAXS }}$ by a renormalisation contribution depending on the characteristic structural length scales $d_{T S}{ }^{7}$ However, it is possible to relate the $\kappa_{\text {SAXS }}$ values to the $\kappa$ from NSE experiments and a respective theoretical $\kappa^{\text {theo }}$ is obtained from the SAXS results using the expression

$$
\frac{\kappa^{\text {theo }}}{k T}=\frac{\kappa_{S A X S}}{k T}+\frac{3}{4 \pi} \ln \left(\frac{d_{T S}}{2 l_{c}}\right)
$$

$l_{c}$ is the thickness of the surfactant monolayer. Both results are also shown in Table 2. A thickness parameter $l_{c}$ in the range 16-25 $\AA$ was estimated using Tanford's formula for the length of carbon chain and dimensions of a glucose group computed in. ${ }^{40}$ The mean value of $\kappa^{\text {theo }}$ obtained based on this is rather small even taking into account a relative uncertainty of 20 percent, estimated due to the approximations made in the calculations. Additionally, the technical grade surfactant is of low efficiency leading to a rather small domain size and the obtained small values for $\kappa^{\text {theo }}$. However, from the SAXS measurements no temperature effect on the structural parameters can be obtained.

\subsection{Collective dynamics as seen with dynamic light scattering}

The normalized intensity time autocorrelation function $g^{2}(q, \tau)$ of five identically composed microemulsion samples was measured at six $q$ values in the temperature range between $278 \mathrm{~K}$ and $302 \mathrm{~K}$. In Fig. 3 three examples of $g^{2}(q, \tau)$ are shown for absolute values of the scattering vector of $q=$ $0.012 \mathrm{~nm}^{-1}, q=0.019 \mathrm{~nm}^{-1}$ and $q=0.027 \mathrm{~nm}^{-1}$ measured at a temperature of $298 \mathrm{~K}$. The relaxation rate distributions resulting from the CONTIN analysis are shown in the same figure. The CONTIN analysis revealed two well separated contributions to the relaxation rate distribution in the order of $10^{3} \mathrm{~s}^{-1}$ and $10^{4} \mathrm{~s}^{-1}$. The maximum of the faster relaxation mode is 5 to 10 times smaller than that of the slower relaxation mode. Following the discussion in the literature on related systems, a hydrodynamic origin of the dynamics in the respected $q$-range was assumed. Therefore, graphs (a) and (b) of Fig. 4 show the relaxation rates $\Gamma$ of the two observed relaxation modes as a function of the square of the wave vector $q$ for five sample temperatures. The symbol size represents the error of the measurements and within this accuracy the slow mode is a linear function of $q^{2}$. While in graph (b) a linear slope is observed this behaviour is difficult to confirm for the data obtained from the faster mode shown in graph (a). However, in both graphs increasing sample temperature corresponds to an increase in relaxation rates. The linearity in graph (b) confirms the hydrodynamic nature of the observed relaxation process.
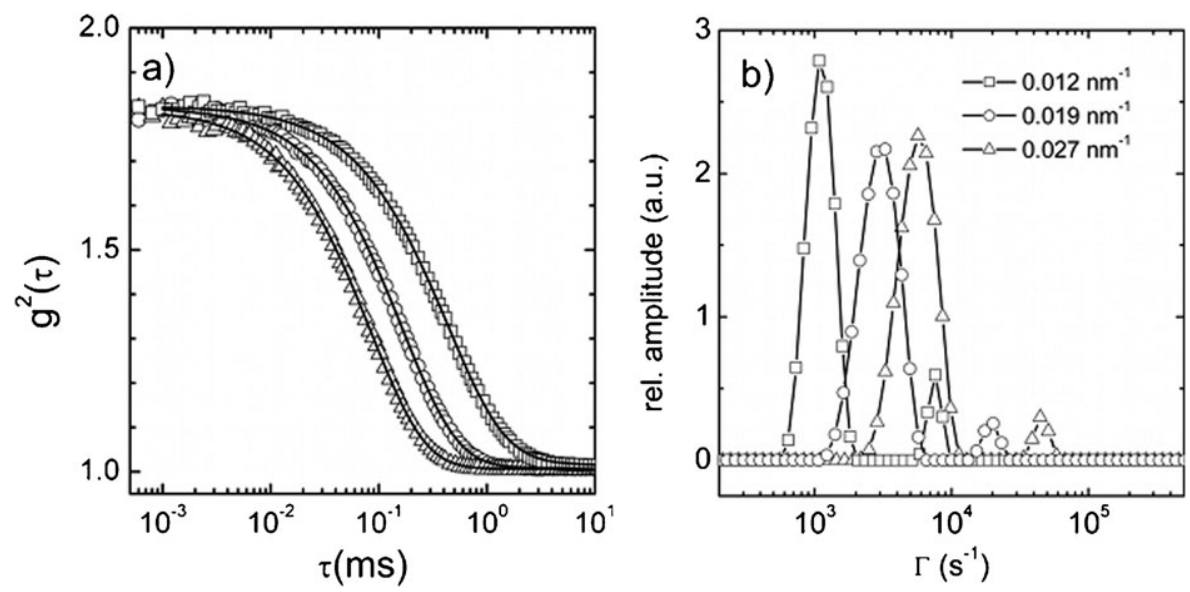

Fig. 3 The left graph shows three representative intensity autocorrelation function measured at $298 \mathrm{~K}$ and $q$ values of 0.012 nm ${ }^{-1}(\bullet)$, $0.019 \mathrm{~nm}^{-1}(\bigcirc)$ and $0.027 \mathrm{~nm}^{-1}(\triangle)$, respectively. The right graph shows the corresponding relaxation rate distributions. Two contributions separated by a factor of 10 are distinguishable for all $q$ values. 

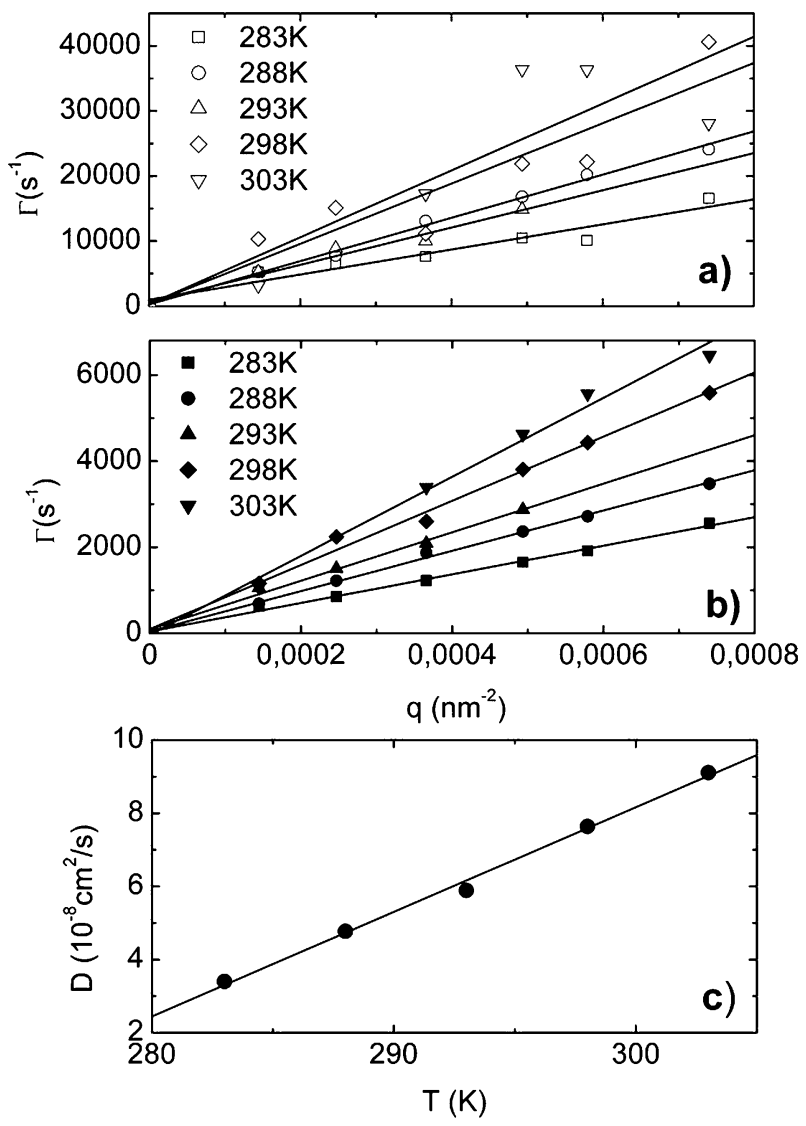

Fig. 4 Results of the CONTIN analysis of the dynamic light scattering measurements on a bicontinuous sample at different sample temperatures. (a) Plot of the mean relaxation rate $\Gamma$ of the fast relaxation mode as function of $q^{2}$. Solid lines are linear fits to the data. (b) Plot of the mean relaxation rate $\Gamma$ of the slower relaxation mode as function of $q^{2}$. Solid lines are linear fits to the data. (c) Temperature dependent diffusion coefficient of the slow relaxation mode calculated according to the dispersion relation $\mathrm{D}=\Gamma / q^{2}$.

From the slope of a linear fit to the data according to the dispersion relation

$$
\Gamma=D q^{2}
$$

the diffusion coefficient related to a slow collective mode of the oil and water phase was obtained for all measured sample temperatures. Although the data obtained from the faster mode shown in Graph (a) deviate partly from the ideal linear slope the same general temperature dependence is observed. In graph (c) of Fig. 4, the diffusion coefficient determined from the slow relaxation mode is shown as a function of the sample temperature. Since a bicontinuous structure consists of randomly oriented interconnected membranes, the measured relaxation rates are averaged values taking into account different physical origins of the relaxation processes within the observed time window. The obtained diffusion coefficient increases from $3.4 \times 10^{-8} \mathrm{~cm}^{2} \mathrm{~s}^{-1}$ at $283 \mathrm{~K}$ up to $9.1 \times$ $10^{-8} \mathrm{~cm}^{2} \mathrm{~s}^{-1}$ at $303 \mathrm{~K}$. A linear relation $\mathrm{D}(\mathrm{T})=0.28576 \times$ (T-271.4) with the units being $[\mathrm{D}]=10^{-8} \mathrm{~cm}^{2} \mathrm{~s}^{-1},[\mathrm{~T}]=\mathrm{K}$ ) was derived. Thus, the diffusion coefficient at any desired temperature can be estimated. Assuming that the diffusion coefficient can be described as

$$
D=D_{0} \exp \left(-E_{A} / k_{\mathrm{B}} T\right)
$$

an activation energy of $14 k_{\mathrm{B}} T$ was estimated.

This energy is approximately three times larger than the activation energy found in a concentrated swollen droplet phase with a characteristic length scale ten times larger than in our bicontinuous structure. ${ }^{16}$ Topological rearrangement by membrane rupture and fusion is an activated process. The characteristic length scale and the high solvent viscosity in our system could explain the comparatively high activation energy in a system where an energy of the order of $k_{\mathrm{B}} T$ induces undulations of the amphiphilic film.

\subsection{Determination of $\boldsymbol{\kappa}$ by combing NSE and DLS}

Neutron spin echo spectroscopy is an ideal method to detect the thermally activated stochastic motions of the amphiphilic interface in microemulsions with a bicontinuous structure $^{7-9,41-46}$ as well as in diluted or cubic droplet phases on a local scale. . $^{28,44-46}$

Also from the theoretical point of view microemulsions are interesting systems and in the context of the present study especially the works by Zilman and Granek are important. Zilman and Granek have investigated the local dynamics of thermally excited undulations of an ensemble of randomly oriented membrane plaquettes. ${ }^{47}$ They derived a stretched exponential form for the intermediate scattering function related to this kind of motion, which is given by

$$
S(q . t) \propto S(q, 0) \exp \left(-\left(\Gamma_{u} \tau\right)^{\beta}\right)
$$

The relaxation rate $\Gamma_{u}$ is expressed by

$$
\Gamma_{u}=0.025 \gamma_{\kappa}\left(\frac{k_{\mathrm{B}} T}{\kappa}\right)^{1 / 2} \frac{k_{\mathrm{B}} T}{\eta} q^{3} .
$$

The relaxation rate shows a linear $q^{3}$ dependence, which is also observed in Zimm polymer dynamics. ${ }^{48}$ The measurement of $\Gamma_{u}$ in a scattering experiment allows the direct determination of the bending elastic constant $\kappa$. The prefactor $\gamma_{\kappa}$ depends on $\kappa$ and $\gamma_{\kappa} \rightarrow 1$ for $\kappa / k_{\mathrm{B}} T \gg 1$. For the

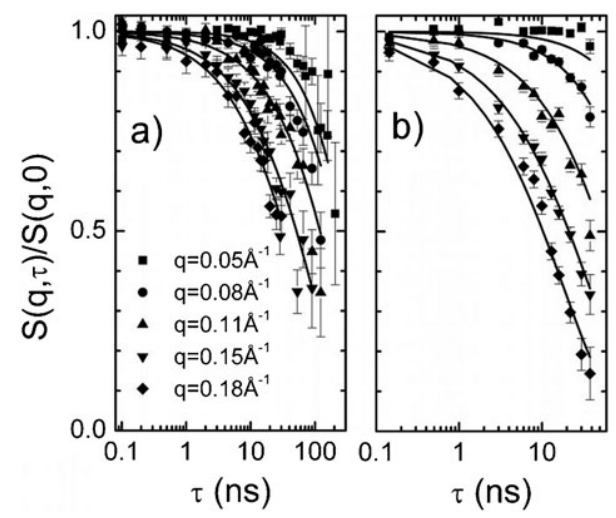

Fig. 5 Normalized intermediate scattering function $\mathrm{S}(q, \tau) / \mathrm{S}(q, 0)$ in semi-logarithmic representation measured for a bicontinuous sample at (a) $283 \mathrm{~K}$ and (b) $298 \mathrm{~K}$ at five $q$-values. The solid lines are fits using eqn (11). 
low $q$-range $q \ll q_{0}$, where $q_{0}$ corresponds to the position of the structure factor peak as obtained from small angle scattering, Nonomura and Ohta studied the dynamics of concentration fluctuations in ternary fluids resulting in hydrodynamic relaxation modes. ${ }^{49}$ In case of bulk contrast samples these hydrodynamic modes contribute to the measured NSE signal. ${ }^{8,9,49}$

A series of NSE measurements was performed with bicontinuous samples of identical composition. The samples were measured in the range $q=0.05 \AA^{-1}$ to $q=0.18 \AA^{-1}$ at temperatures between $283 \mathrm{~K}$ and $303 \mathrm{~K}$. Fig. 5 shows two examples for the obtained experimental data. The image (a) of Fig. 5 contains the normalized intermediate scattering functions $S(q, \tau) / S(q, 0)$ measured at $283 \mathrm{~K}$ and in image (b) on the right the data obtained at $298 \mathrm{~K}$ are plotted. Within the investigated Fourier time window no complete relaxation of $S(q, \tau) / S(q, 0)$ was observed. The low temperature measurement shows significantly slower dynamics than observed at higher temperatures, at a Fourier time of 100 ns at all $q$-values no complete decay is observed. In comparison to this, at $298 \mathrm{~K}$ the intermediate scattering function is down to $0.1-0.2$ at $40 \mathrm{~ns}$ at the highest $q$-value. The solid lines are fits to the data using eqn (11), which takes into account the contribution of the hydrodynamic motion to the decay of the normalized intermediate scattering function. The chosen model is given by

$S(q, \tau) / S(q, 0)=\exp \left(-\Gamma_{D i f f} \tau\right)\left(A+(1-A) \exp \left(-\left(\Gamma_{u} \tau\right)^{\beta}\right)\right.$.

where $\Gamma_{\text {Diff }}$ is the relaxation rate of the collective hydrodynamic modes calculated based on the diffusion coefficient measured by DLS using the dispersion relation in eqn (7). Following previous work on dilute droplet microemulsions ${ }^{29}$ and also on bilayer motion in vesicles ${ }^{10,11}$ the diffusion constants obtained from DLS measurements were included in the data analysis. Therefore, the relaxation rates $\Gamma_{D i f f}$ in eqn (11) were calculated for each temperature and Table 3 summarizes these values.

Undulation motions are taken into account by the rate $\Gamma_{u}$ and $\beta=2 / 3$ is the stretching exponent introduced by the Zilman Granek approach. In cubic microemulsion phases a similar model was successfully used to explain the observed dynamics. ${ }^{8}$ As shown in section 2.5 dynamic light scattering measures the hydrodynamic motions in the bicontinuous microemulsion.

Fig. 6 plots the undulation relaxation rates $\Gamma_{u}$ versus $q^{3}$ for the investigated sample temperatures. The $\Gamma_{u}$ values were obtained from the best results of fitting eqn (11) to the data using a Levenberg-Marquardt algorithm. Within the accuracy of the measurement, at all temperatures the predicted linear

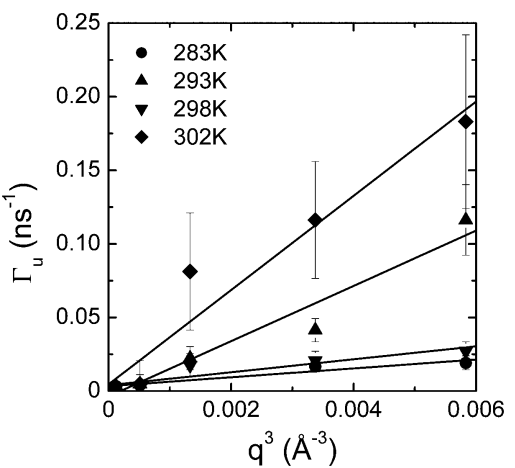

Fig. 6 Undulation relaxation rates $\Gamma_{u}$ as a function of $q^{3}$ for four sample temperatures in the range $283 \mathrm{~K}-302 \mathrm{~K}$. From linear fits to the data (solid lines) the bending elasticity constant $\kappa$ was calculated using eqn (10).

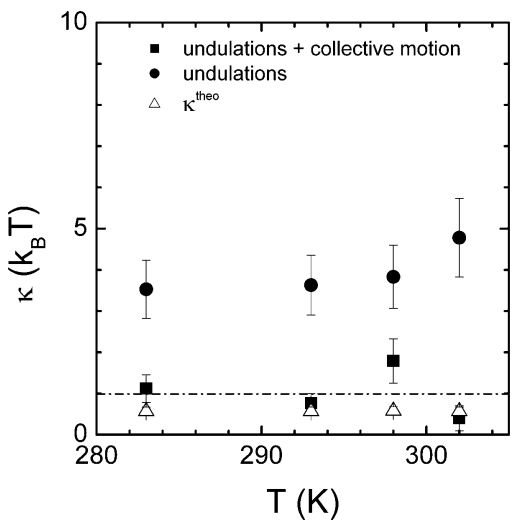

Fig. 7 Bending elastic constant $\kappa$ as a function of sample temperature resulting from an analysis using model 11 , a treatment only taking into account the undulation motion described by eqn (9) and the theoretically from SAXS data expected values $\kappa^{\text {theo }}$.

relation between $\Gamma_{u}$ and $q^{3}$ can be confirmed and according to eqn (10) from the slope of a linear fit to the data the bending elasticity constant $\kappa$ was calculated for each temperature. In Fig. 7 all results for $\kappa$ are summarized and plotted as a function of sample temperature. The absolute values are of the order of $1 k_{\mathrm{B}} T$ for all investigated temperatures if collective motions are included in the analysis. No significant change of $\kappa$ as a function of temperature could be observed from the data. Additionally, the bending elasticities were calculated without including the contribution of the collective hydrodynamic motions in the fitting routine and comparatively large values were obtained also shown in Fig. 7. Within the investigated temperature range no significant change of the bending elasticity constants obtained from static and dynamic

Table 3 Temperature dependence of the diffusion coefficient D measured by dynamic light scattering and the calculated relaxation rates $\Gamma_{\text {diff }}$

\begin{tabular}{llllll}
\hline & & & & \\
\cline { 3 - 6 } & & & & \\
diff $(1 / \mathrm{ns})$ & $q=0.08^{-1}$ & $q=0.11^{-1}$ & $q=0.15^{-1}$ \\
\hline 283 & $\mathrm{D} / 10^{-8} \mathrm{~cm}^{2} \mathrm{~s}^{-1}$ & $q=0.05 \AA^{-1}$ & $2.5 \times 10^{-3}$ & $4.7 \times 10^{-3}$ & $8.7 \times 10^{-3}$ \\
293 & 3.87 & $9.7 \times 10^{-4}$ & $3.8 \times 10^{-3}$ & $7.1 \times 10^{-3}$ & $1.3 \times 10^{-2}$ \\
298 & 5.89 & $1.5 \times 10^{-3}$ & $4.9 \times 10^{-3}$ & $9.2 \times 10^{-3}$ & $1.7 \times 10^{-2}$ \\
302 & 7.64 & $1.9 \times 10^{-3}$ & $5.8 \times 10^{-3}$ & $1.1 \times 10^{-2}$ & $2.1 \times 10^{-2}$ \\
\hline
\end{tabular}


measurements was found. A similar result was obtained from investigations in a ternary $\mathrm{AOT}-\mathrm{D}_{2} \mathrm{O}$-Toluene droplet microemulsion, where the consistency of the bending elasticity was proofed using NSE and SANS measurements over a wide temperature range. ${ }^{50}$

The bending elasticity constants obtained from the combination of DLS and NSE are significantly larger than the renormalized and the bare values $\kappa_{\text {SAXs }}$ and $\kappa^{\text {theo }}$ extracted from the static structure factor of the bicontinuous structure. This calculation is based on the characteristic length scales in the system. NSE measurements are governed by dissipative processes in the system, which are mediated by the viscosity of the involved liquids. In the simplest case, the oil and the water domain have the same viscosity and the approximations made in the Zilman-Granek approach are valid. In the present system the viscosity is asymmetric. Hence, for the calculations the dynamic viscosity of RME was used to estimate the viscosity of the medium surrounding the undulating membranes. This choice seems to be justified, because the dynamic viscosity of RME is by a factor of 5 to 10 larger than the viscosity of $\mathrm{D}_{2} \mathrm{O}$ and therefore, assumed to be the main contribution to the energy dissipation in the system leading to larger results of $\kappa$. Furthermore, the undulation of the membrane can strongly increase the viscosity, as theoretically explained in the literature ${ }^{51,52}$ and experimentally shown in NSE experiments. ${ }^{41}$

\section{Conclusions}

In this contribution an environmentally compatible microemulsion system consisting of the four components waterRME- $C_{12 / 14} G_{1.3}$-Pentanol was under investigation using quasi-elastic scattering methods. The aim was to measure the bending elastic constant $\kappa$ over a wide temperature range. In contrast to the $C_{\mathrm{i}} \mathrm{E}_{j}$ surfactants, the present system allows to address this problem, due to the low temperature dependence of the phase behaviour in alkyloligoglucoside based microemulsions.

The dynamics in the bicontinuous phase of the investigated microemulsion system was studied in a comparatively large temperature range and over a wide $q$-range. The relaxation time window obtained by the combination of dynamic light scattering and neutron spinecho spectroscopy covers 9 orders of magnitude. From $q$-dependent dynamic light scattering measurements diffusion coefficients in the range of $D=3.4 \times 10^{-8} \mathrm{~cm}^{2} \mathrm{~s}^{-1}$ to $D=9.1 \times 10^{-8} \mathrm{~cm}^{2} \mathrm{~s}^{-1}$ for the collective motions in the bicontinuous structure were found. These results were then used to calculate relaxation rates for these collective motions in the $q$-range explored by neutron spinecho spectroscopy assuming that the linear dispersion in $q^{2}$ is still valid when $q$ reaches the range around the position of the maximum of the static structure factor $q_{\max }$. Including these predicted relaxation rates in the analysis of the NSE data, reasonable values for the bending elastic constant $\kappa$ were obtained. Within the precision of the presented experiments, the bending elastic constant is independent of temperature in the investigated interval. However, at present only a low number of studies was done on sugar surfactant based microemulsions and more combined DLS and NSE experiments are required to gain deeper insight into the interplay of long range collective motions and the local undulations related to the bending elastic constant $\kappa$ of the amphiphilic interface.

\section{References}

1 J. Reimer, O. Söderman, T. Sottmann, K. Kluge and R. Strey, Langmuir, 2003, 19, 10692-10702.

2 C. Stubenrauch, B. Paeplow and G. H. Findenegg, Langmuir, 1997, 13, 3652-3658.

3 C. Stubenrauch and G. H. Findenegg, Langmuir, 1998, 14, 6005-6012.

4 C. Stubenrauch, Curr. Opin. Colloid Interface Sci., 2001, 6, $160-170$.

5 A. Stradner, O. Glatter and P. Schurtenberger, Prog. Colloid Polym. Sci., 2000, 115, 10-14.

6 F. Mezei, Neutron Spin Echo, Berlin, 1980, pp. 3-26.

7 O. Holderer, H. Frielinghaus, D. Byelov, M. Monkenbusch, J. Allgaier and D. Richter, J. Chem. Phys., 2005, 122, 094908/1-8.

8 O. Holderer, H. Frielinghaus, M. Monkenbusch, J. Allgaier, D. Richter and B. Farago, Eur. Phys. J. E, 2007, 22, 157-161.

9 M. Mihailescu, M. Monkenbusch, H. Endo, J. Allgaier, G. Gompper, D. Richter, B. Jakobs, T. Sottmann and B. Farago, J. Chem. Phys., 2001, 115, 9563-9577.

10 L. R. Arriaga, I. Lopez-Montero, F. Monroy, G. O. Gil, B. Farago and T. Hellweg, Biophys. J., 2009, 96, 3629-3637.

11 L. R. Arriaga, I. Lopez-Montero, F. Monroy, G. O. Gil, B. Farago and T. Hellweg, Phys. Rev. E, 2009, 80, 031908.

12 S. Wellert, H.-J. Altmann, A. Richardt, A. Lapp, P. Falus, B. Farago and T. Hellweg, Eur. Phys. J. E, 2010, 33, DOI: 10.1140/epje/i2010-10668-1.

13 T. Morkved, B. R. Chapman, F. Bates, T. Lodge, P. Stepanek and K. Almdal, Faraday Discuss., 1999, 112, 335-50.

14 A. Shukla, H. Graener and R. Neubert, Langmuir, 2004, 20, $8526-8530$.

15 E. Freyssingeas, F. Nallet and D. Roux, Langmuir, 1996, 12, 6028-6035.

16 U. Peter, D. Roux and A. K. Sood, Phys. Rev. Lett., 2001, 86, $3340-3343$.

17 M. Maugey and A. M. Bellocq, Langmuir, 2001, 17, 6740-6742.

18 J. Oizumi, Y. Kimura, K. Ito and R. Hayakawa, Mol. Cryst. Liq. Cryst., 1997, 303, 63-71.

19 M. Teubner and R. Strey, J. Chem. Phys., 1987, 87, 3195-3200.

20 S. H. Chen, S. L. Chang, R. Strey and P. Thiyagarajan, J. Phys.. Condens. Matter, 1991, 3, F91-F107.

21 O. Glatter, R. Strey, K.-V. Schubert and E. W. Kaler, Ber. Bunsenges. Phys. Chem., 1996, 100, 323-335.

22 J. Brunner-Popela, R. Mittelbach, R. Strey, K.-V. Schubert, E. W. Kaler and O. Glatter, J. Chem. Phys., 1999, 110, 10623-10632.

23 P. Pieruschka, S. A. Safran and S. T. Marcelja, Phys. Rev. E, 1995, 52, 1245-1247.

24 G. Gompper and D. M. Kroll, Phys. Rev. Lett., 1998, 81, 2284-2287.

25 Y.-C. Yang, J. Baker and J. Ward, Chem. Rev., 1992, 92, $1729-1743$.

26 J. Gäb, M. Melzer, K. Kehe, S. Wellert, T. Hellweg and M.-M. Blum, Anal. Bioanal. Chem., 2010, 396, 1213-1221.

27 S. A. Safran, Adv. Phys., 1999, 48, 395-448.

28 T. Hellweg and D. Langevin, Phys. Rev. E, 1998, 57, 6825-6834.

29 T. Hellweg, M. Gradzielski, B. Farago and D. Langevin, Colloids Surf., A, 2001, 183-185, 159-169.

30 S. Wellert, M. Karg, H. Imhof, A. Steppin, H.-J. Altmann, M. Dolle, A. Richardt, B. Tiersch, J. Koetz, A. Lapp and T. Hellweg, J. Colloid Interface Sci., 2008, 325, 250-258.

31 CRC Handbook of Chemistry and Physics, ed. R. C. Weast, CRC Press, Inc., Boca Raton, Florida, 64th edn, 1983.

32 M. Kahlweit and R. Strey, J. Phys. Chem., 1988, 92, 1557-1563.

33 A. Bergmann, D. Orthaber, G. Scherf and O. Glatter, J. Appl. Crystallogr., 2000, 33, 869-875.

34 D. Orthaber, A. Bergmann and O. Glatter, J. Appl. Crystallogr., 2000, 33, 218-223.

35 S. W. Provencher, Comput. Phys. Commun., 1982, 27, 213-217. 
36 M. Monkenbusch, Neutron News, 1997, 8, 25.

37 M. Monkenbusch, R. Schatzler and D. Richter, Nucl. Instrum. Methods Phys. Res., Sect. A, 1997, 399, 301-323.

38 J. Kohlbrecher, SASfit: A program for fitting simple structural models to small angle scattering data, Paul Scherrer Institut, Laboratory for Neutron Scattering, CH-5232 Villigen, Switzerland, 2008.

39 G. Gompper, H. Endo, M. Mihailescu, J. Allgaier, M. Monkenbusch, D. Richter, B. Jakobs, T. Sottmann and R. Strey, Europhys. Lett., 2001, 56, 683-689.

40 R. Netrabukkana, K. Lourvanij and G. L. Rorrer, Ind. Eng. Chem. Res., 1996, 35, 458-464.

41 S. Komura, T. Takeda, Y. Kawabata, S. K. Gosh, H. Seto and M. Nagao, Phys. Rev. E, 2001, 63, 41402-41411.

42 S. Komura, T. Takeda, Y. Kawabata, S. K. Gosh, H. Seto and M. Nagao, Eur. Phys. J. E, 2001, 5, 329-336.
43 M. Monkenbusch, O. Holderer, H. Frielinghaus, D. Byelov and D. Richter, J. Phys.: Condens. Matter, 2005, 17, S2903-S2909.

44 B. Farago, Phys. B, 1996, 226, 51-55.

45 T. Hellweg and D. Langevin, Phys. A, 1999, 264, 370-387.

46 T. Hellweg, A. Brûlet and T. Sottmann, Phys. Chem. Chem. Phys., 2000, 2, 5168-5174.

47 A. G. Zilman and R. Granek, Phys. Rev. Lett., 1996, 77, 4788-4791.

48 S. S. Sorlie and R. Pecora, Macromolecules, 1988, 21, 1437-1449.

49 M. Nonomura and T. Ohta, J. Chem. Phys., 1999, 110, 7516-7523.

50 T. Spehr, B. Frick, I. Grillo, P. Falus, M. Müller and B. Stühn, Phys. Rev. E, 2009, 79, 0314041-03140412.

51 N. L. Yamada, T. Takeda, K. Kato, M. Nagao and H. Seto, J. Phys. Soc. Jpn., 2005, 74, 875-877.

52 N. Gov, A. G. Zilman and S. Safran, Phys. Rev. E, 2004, 70, 0111041-01110410.

53 M. Kahlweit and R. Strey, Angew. Chem., 1985, 97, 655-669. 